\title{
Coupled blind signal separation and spectroscopic database fitting of the mid-infrared PAH features ${ }^{\star}$ (Corrigendum)
}

\author{
M. J. F. Rosenberg ${ }^{1,2}$, O. Berné ${ }^{1}$, C. Boersma ${ }^{3}$, L. J. Allamandola ${ }^{3}$, and A. G. G. M. Tielens ${ }^{1}$ \\ 1 Sterrewacht Leiden, Universiteit Leiden, Niels Bohrweg 2, 2333 CA Leiden, The Netherlands \\ e-mail: rosenberg@strw. leidenuniv.nl \\ 2 The International Space University, Parc d'Innovation, 1 rue Jean Dominique Cassini, 67400 Illkirch Graffenstaden, France \\ 3 NASA Ames Research Center, Space Science Division, Mail Stop 245-6, Moffett Field, CA 94035, USA \\ e-mail: [Louis.J.Allamandola;Christiaan.Boersma]@nasa.gov
}

A\&A 532, A128 (2011), DOI: 10.1051/0004-6361/201016340

Key words. astrochemistry - infrared: ISM - ISM: lines and bands - photon-dominated region (PDR) - Errata, addenda

\section{Erratum}

The original paper contained erroneous data in Fig. 10. The correct figure is presented here. None of the conclusions are substantially affected.

Figure 10 displays a linear correlation between the $[11.0] /[11.2] \mu \mathrm{m}$ PAH emission line ratio and the $[6.2] /[11.2] \mu \mathrm{m}$ ratio. To measure the $[6.2] /[11.2] \mu \mathrm{m}$ ratio, the low-resolution IRS spectra were used to measure the flux of the $6.2 \mu \mathrm{m}$ feature, and the high-resolution spectra to measure the $11.2 \mu \mathrm{m}$ band flux. As noted in the text, this was chosen because the [11.0] $\mu \mathrm{m}$ contribution cannot be differentiated from the [11.2] $\mu \mathrm{m}$ emission using the low-resolution module. However, upon inspection, we have found that the low-resolution and high-resolution IRS modules are not calibrated correctly with respect to each other. There is on average a factor of $\sim 2$ difference in the absolute intensity between low and high-resolution spectra in the 9-14 $\mu \mathrm{m}$ range.

Although the exact origin of this calibration problem is beyond the scope of this paper, we believe it comes from the extended nature of the emission in NGC 7023-NW. This PDR has many bright filaments and complex spatial structures that are unresolved by the IRS instrument. The difference in aperture size between the low and high-resolution modes, namely $\left(1.8^{\prime \prime}\right)^{2}$ for high and $\left(2.3^{\prime \prime}\right)^{2}$ for low, could account for this factor of two difference between the modules. Comparing IRS low-resolution spectral mapping with IRAC $8 \mu \mathrm{m}$ image also shows a large discrepancy in the observed integrated intensities.
Once this cross-calibration problem between the high and low resolution modules of IRS is apparent, we must alter our method of measuring the ratios. For the $[6.2] /[11.2] \mu \mathrm{m}$ ratio, it is necessary to measure both the [6.2] and [11.2] $\mu \mathrm{m}$ bands with the same module (i.e. IRS-SL), so the calibration offset is normalized. However, this presents the problem of the $11.0 \mu \mathrm{m}$ $\mathrm{PAH}^{+}$contamination, since the $11.0 \mu \mathrm{m}$ contribution cannot be resolved in the low-resolution mode. This can be corrected for using the high-resolution $[11.0] /[11.2] \mu \mathrm{m}$ ratio. The integrated intensity in the 11.2 band, $I_{11.2}$, can be expressed independantly of the $\mathrm{SH} / \mathrm{SL}$ calibration by

$I_{11.2}=I_{11.2}^{\mathrm{SL}} \times\left(1-I_{11.0}^{\mathrm{SH}} / I_{11.2}^{\mathrm{SH}}\right)$.

A new plot has been created using the new $I_{11.2}$ and is shown in Fig. 1 . The low signal-to-noise ratio points $(\leq 25 \%$ of maximum flux) were removed and the remaining points were fit with a least squares linear regression. The revised empirical relation is

$\frac{[11.0 \mu \mathrm{m}]}{[11.2 \mu \mathrm{m}]}=0.084 \times\left(\frac{[6.2 \mu \mathrm{m}]}{[11.2 \mu \mathrm{m}]}\right)-0.06$.

We note that this relation does not go through $(0,0)$. This implies that there is $6.2 \mu \mathrm{m}$ emission even if there is no $11.0 \mu \mathrm{m}$ band. Recalling that the $6.2 \mu \mathrm{m}$ band may have a (weak) contribution from neutral PAHs, while the $11.0 \mu \mathrm{m}$ band is "pure" cation, we suggest that the $11.0 \mu \mathrm{m}$ band may be a better tracer of the presence of PAH cations in space. Further studies will have to validate this statement.

* This work is based on observations made with the Spitzer Space Telescope, which is operated by the Jet Propulsion Laboratory, California Institute of Technology under a contract with NASA. 


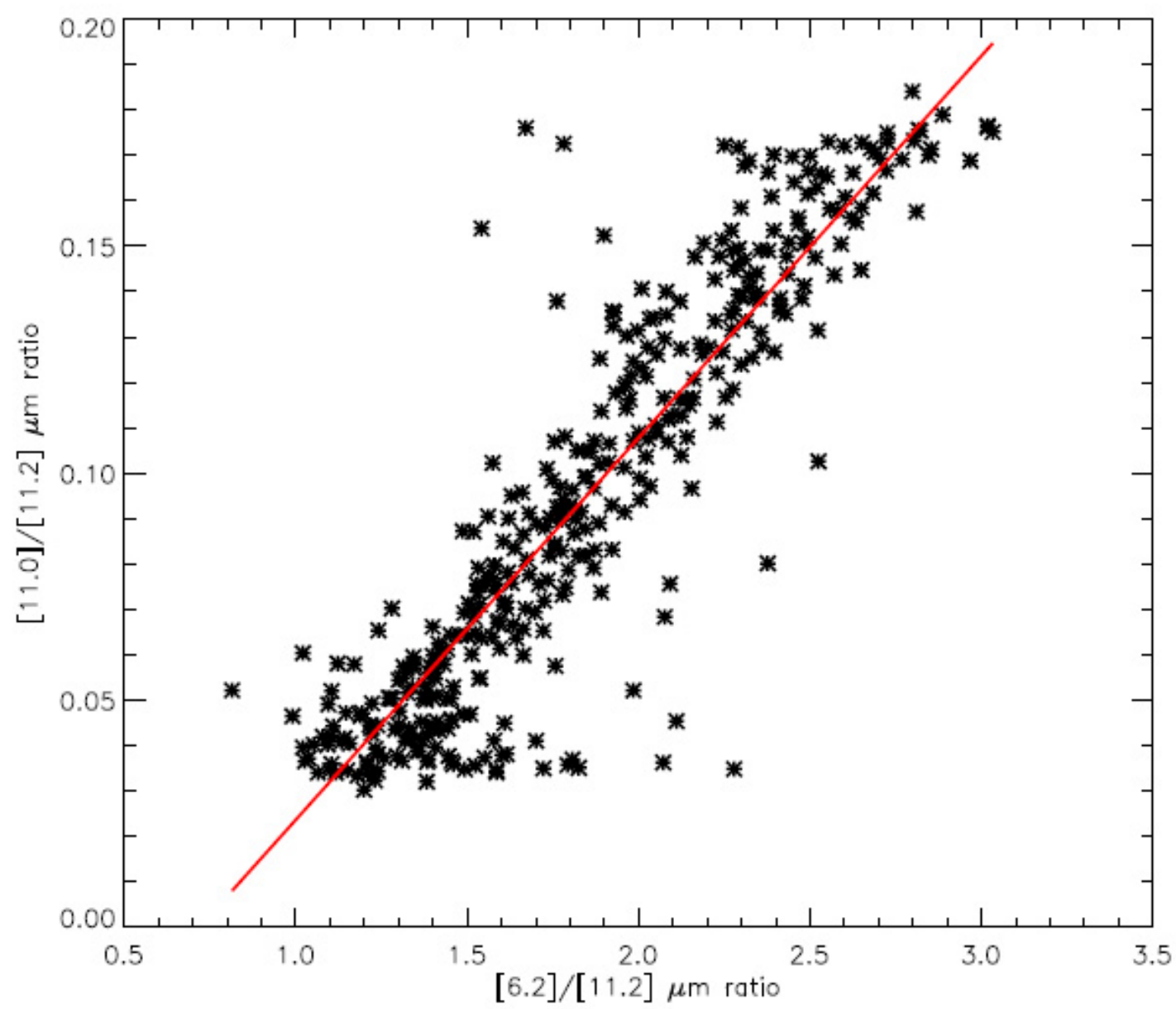

Fig. 1. The $[6.2] /[11.2] \mu \mathrm{m}$ vs. the $[11.0] /[11.2] \mu \mathrm{m}$ PAH band integrated intensity ratio in NGC 7023-NW. The $11.2 \mu \mathrm{m}$ and $11.0 \mu \mathrm{m}$ measurements were made using the IRS-SH observations, while the [6.2]/[11.2] $\mu \mathrm{m}$ ratio was measured using the IRS-SL observations (see text for details). The instrumental error is comparable to the symbol size. 